\title{
Les évadés de l'île de Pâques. Loin du Chili, vers Tahiti (1944-1958), de Marie-Françoise PETEUIL
}

Hélène Guiot

\section{OpenEdition}

\section{Journals}

Édition électronique

URL : http://journals.openedition.org/jso/5850

DOI : 10.4000/jso.5850

ISSN : $1760-7256$

Éditeur

Société des océanistes

\section{Édition imprimée}

Date de publication : 30 juin 2009

Pagination : 162-163

ISBN : 978-2-85430-024-6

ISSN : 0300-953x

\section{Référence électronique}

Hélène Guiot, "Les évadés de lîle de Pâques. Loin du Chili, vers Tahiti (1944-1958), de Marie-Françoise PETEUIL », Journal de la Société des Océanistes [En ligne], 128 I janvier-juin 2009, mis en ligne le 30 juin 2009, consulté le 24 septembre 2020. URL : http://journals.openedition.org/jso/5850 ; DOI : https:// doi.org/10.4000/jso.5850 
étant sans doute moins sensible à ce style graphique qu'à celui présent dans les autres.

Quoi qu'il en soit, on ne peut que saluer les auteurs de ces volumes qui mettent à la portée du plus grand nombre de nombreux aspects de la culture kanak.

Isabelle LEBLIC, CNRS-LACITO, Villejuif

PÉTEuIL Marie-Françoise, 2004. Les évadés de l'île de Pâques. Loin du Chili, vers Tahiti (1944-1958), Paris, L'Harmattan, 270 p.,bibliogr., cartes et ill.

Après une année 2008 riche en expositions sur la Polynésie (Bounoure, dans ce numéro : pp. 147-151), dont deux consacrées à Rapa Nui, l'île de Pâques, il est essentiel de lire, voire de relire, l'ouvrage de MarieFrançoise Peteuil, Les évadés de l'île de Pâques. Il n'est pas question ici de l'art sophistiqué des anciens Pascuans, non plus que des moai, encore moins de prétendus mystères. M.-F. Peteuil s'inscrit dans une recherche trop rarement développée en France, l'histoire contemporaine des sociétés du Pacifique, et la lecture de son ouvrage ouvre une fenêtre sur l'histoire du $\mathrm{Xx}^{\mathrm{e}}$ siècle, mal connue, voire cachée, de l'île de Pâques : à huit reprises, des Pascuans organisèrent des voyages d'évasion pour fuir leur propre terre...

Le récit de Marie-Françoise Peteuil aurait pu s'apparenter à tant d'autres décevantes chroniques d'Européens qui découvrent la Polynésie. Heureusement, dès les premières lignes, elle nous entraîne d'un ton alerte et non dénué d'humour, sur son chemin de prof (sic !) qui, « tentant d'enseigner les mathématiques républicaines à des collégiens de Tahiti », découvre, bien au-delà des clichés, la profondeur historique d'une population et les événements $\mathrm{du} \mathrm{Xx}^{\mathrm{e}}$ siècle qui s'y rattachent. Le ton léger des premières lignes, à l'image des représentations qu'ont les Occidentaux sur la Polynésie, leurs habitants et leur histoire, ne préfigure absolument pas l'histoire grave que nous dévoilent les recherches de l'auteur. Tout est là, dans l'opposition entre ce que nous connaissons, ou ce qui nous est raconté, de l'île de Pâques et la réalité des faits qu'a minutieusement reconstituée M.-F. Peteuil.

Présenté comme une enquête, l'ouvrage s'articule en six parties, auxquelles s'ajoutent des annexes et une bibliographie. Dans la première, au titre évocateur de "L'île de Pâques est habitée ! ", l'auteur relate sa découverte de la réalité pascuane. Lors de son séjour, elle entend parler pour la première fois de ces voyages d'évasion et décide d'en savoir plus.

Ainsi, dans la deuxième partie, M.-F. Peteuil plonge dans la bibliographie des récits sur l'île de Pâques, à la recherche de toute donnée en rapport avec ces navigations, ces départs furtifs pour fuir la misère et l'enfermement instauré par la colonisation chilienne. Elle opère une lecture critique d'Heyerdahl, de Mazière, d'Englert, propose une chronologie sur le passage des Occidentaux à l'île de Pâques, de Roggeveen en 1722 aux évasions des années 1950, en passant par James
Cook et Beechey, sans oublier la terrible année 1862 durant laquelle « 1500 Pascuans furent déportés [vers les exploitations agricoles du Pérou], soit la moitié de la population totale. Parmi les captifs, l'ensemble de la classe des prêtres et des initiés, le roi Kamakoi et son fils Maurata » (p. 41). Une année plus tard, suite à l'intervention du gouvernement français via $\mathrm{M}^{\mathrm{gr}}$ Jaussen à Tahiti et avec l'aide des Anglais, le Pérou est sommé de rapatrier les prisonniers. Une douzaine seulement parvint jusqu'à Rapa Nui et y introduit la variole. " La population de l'île fut alors décimée. L'équilibre économique et social était rompu » (p. 41).

S'ensuivirent l'évangélisation en 1864, les passages de l'aventurier français Dutrou-Bornier, l'installation de Tahitiens par la société commercial Brander, et l'annexion chilienne en 1888. Rapa Nui devint une terre d'élevage de moutons. La population fut tenue de se rassembler à Hanga Roa, village principal, et l'eau rationnée au profit du bétail (p. 45). L'auteur décrit ensuite l'existence carcérale des Pascuans sur leur propre terre, les lois dictatoriales, les vexations, le travail obligatoire jusqu'en 1966. Mais des révoltes sont connues depuis le début $\mathrm{du} \mathrm{xx}^{\mathrm{e}}$ siècle, relatées notamment dans l'ouvrage de K. Routledge à Rapa Nui en 1914.

Marie-Françoise Peteuil poursuit donc ses enquêtes «tous azimuts »: bibliothèques, Internet, contacts auprès des chercheurs spécialistes du sujet, Pascuans d'Europe, du Chili et de Rapa Nui où elle retourne, pour " une approche humaine, celle des rencontres et de la parole échangée » (p. 83). Elle parvient ainsi à reconstituer huit voyages d'évasions dont elle nous livre la chronologie et le récit avec précision et sensibilité, dans la troisième partie.

La première de ces expéditions se situe en 1944 et la dernière en 1958. Toutes ont pour destination projetée l'île de Tahiti. Poussés par la nécessité de fuir le joug chilien, voire de braver l'autorité, certains souhaitent faire entendre leurs revendications politiques afin que Rapa Nui soit placée sous administration française. Pour cela, les navigateurs, tous des hommes, embarquent sur de petits bateaux construits en cachette, ou volés aux Chiliens, souvent aidés par la population, et sans instrument de navigation, s'appuient sur les techniques traditionnelles de navigation océanienne, basées sur de nombreuses et minutieuses connaissances astronomiques, et sur l'observation des phénomènes naturels (voir, à ce sujet, Guiot, 2003; Peteuil, 2003).

La quatrième expédition, en 1954, se termine aux Tuamotu. Les trois navigateurs embarqués furent rapatriés à Tahiti où ils délivrèrent leur demande : une pétition de nombreux Pascuans qui demandaient à la France un statut de protectorat. Mais leur démarche fut totalement ignorée, voire raillée, et seule la réussite de la traversée maritime reste dans les mémoires. À Rapa Nui, elle provoqua d'ailleurs « une véritable épidémie d'évasions » (p. 117), de 1955 à 1958. Pour cette dernière, l'auteur rétablit une réalité révoltante. Sept Pascuans enfermés dans la léproserie de l'île ${ }^{5}$ tentèrent

5. La maladie avait atteint Rapa Nui dès la fin du XIX ${ }^{\mathrm{e}}$ siècle et une léproserie fut construite au début du siècle suivant, à l'écart du village, tenue par des religieuses (p. 144). 
deux évasions. Lors de la seconde, ils périrent en mer. Mais loin d'être « la tentation de moribonds pour une dernière aventure magnifique, c'est bien plutôt l'acte d'hommes en rébellion, arrêtés, enfermés, afin de sortir et de dénoncer la réalité carcérale de la lèpre à Rapa Nui, et espérer y mettre un terme » (p. 148). En effet, au fil des années, cette maladie venue de l'extérieur devint un prétexte pour les autorités pour maintenir les Pascuans isolés du monde, puis pour tenir enfermés tous ceux qui furent déclarés lépreux et «tous les rebelles devinrent des lépreux en puissance » (pp. 147148).

Avec la quatrième partie de l'ouvrage, nous suivons l'insurrection de 1964, menée d'une main de maître par Alfonso Rapu, qui aboutit à la promulgation, en 1966, d'une loi qui instaura l'égalité des droits entre les Pascuans et les autres citoyens chiliens.

Dans son dernier chapitre, « Le rire des Pascuans », Marie-Françoise Peteuil nous livre quelques-unes des paroles recueillies au cours de ses entretiens avec les Pascuans de Rapa Nui et d'ailleurs. Ce rire et ses paroles, comme pour lutter contre le silence et l'oubli, montrent les Pascuans, génération après génération, luttant pour prendre en main leur présent et l'avenir de leurs enfants et maintenir la vigueur de leur culture.

Si l'ethnologue Grant McCall avait déjà mentionné en quelques lignes les voyages d'évasion dans son ouvrage de 1980, puis plus longuement en 1997, Marie-Françoise Peteuil nous offre dans Les évadés de l'île de Pâques, la première étude largement documentée, contextualisée et humaine sur cette période, inconnue du public, de l'histoire contemporaine de l'île de Pâques. Un ouvrage essentiel.

\section{RÉFÉRENCES CITÉES}

Guiot Hélène, 2003. Pirogues, reflets de la Polynésie, Paris, Société des Océanistes, Dossier de la Société des Océanistes.

McCall Grant, 1980. Rapa Nui. Traditions and survival in Easter island, Honolulu, University of Hawai'i Press.

—, 1997a. Rapanui Wanderings: Diaspora from Easter Island, Université du Nouveau Mexique.

—, 1997b. Riro, Rapu, Rapa Nui, Rapa Nui Journal 11, 3, pp. 112-122.

PÉTEuIL Marie-Françoise 2003. Ciel d'îles, Journal de la Société des Océanistes 116, pp. 14-24.

Routledge Katherine, 1920 (2e éd.). The Mystery of Easter Island, London, Sifton Praed and Co.

Hélène GuIOT, ArScAn 7041, Nanterre

Martin Stéphane (éd.), 2009. Mangareva, Panthéon de Polynésie, Paris, Somogy-Musée du quai Branly, 80 p., bibliogr., cartes, 46 ill. couleur.

Le musée du quai Branly a eu l'excellente idée de réunir, le temps d'une exposition (3 février-10 mai 2009), les principales sculptures ayant échappé aux autodafés déclenchés par les missionnaires picpuciens à Mangareva, soit une petite dizaine, complétées par une poignée d'objets « ethnographiques » et quelques documents européens. L'ensemble doit ensuite être présenté au musée de Tahiti et des îles (24 juin-24 septembre 2009), à défaut de Mangareva comme il eût été légitime. À Paris, en dépit d'une présentation austère et d'un éclairage parfois insuffisant ou mal réglé, les visiteurs ont eu l'occasion unique de comparer ces pièces venues de musées éloignés ou de collections missionnaires peu accessibles et de les examiner sous leurs principaux angles, grâce à des vitrines judicieusement disposées. Dans sa préface au catalogue de l'exposition, Stéphane Martin parle de « juste retour des choses », à propos des prêts consentis pour cette manifestation par les musées missionnaires légataires d'une histoire accablante. L'expression s'appliquerait tout aussi bien à l'intérêt enfin marqué par le principal musée français d'arts « premiers » à une civilisation et à des insulaires dont ce pays-ci ne s'est guère soucié jusqu'à l'interruption des essais nucléaires dans le Pacifique et à l'examen très récent et toujours en cours de leurs séquelles.

Principalement dévolu aux arts et à la culture d'avant les missionnaires, le catalogue ne dit à peu près rien de la condition des habitants actuels de Mangareva et du reste des Gambier, ce dont les lecteurs peuvent s'informer par ailleurs, on en conviendra. On ne sait si c'est la même considération, ou des raisons d'économie, ou quelque diktat de responsables commerciaux qui ont retenu de munir les principales contributions de ce petit volume de références qui auraient à la fois fondé leurs assertions et permis à un public de plus en plus large d'approfondir ses connaissances. Quoi qu'il en soit, l'introduction de Tara Hiquily (pp. 8-14) et son essai sur « la représentation humaine à Mangareva et dans les autres îles de Polynésie » (pp. 58-69), comme celui de Jean-Marc Pambrun ( Des dieux, des plantes et des constellations », pp. 71-77) ne sont réellement intelligibles ou appréciables que des connaisseurs ayant en tête ou sous les yeux les textes classiques sur Mangareva et pouvant démêler, dans tel paragraphe, ce qui revient à Beechey, à Laval ou à Te Rangi Hiroa, et ce qui relève des hypothèses de leur citateur. L'estimation de la population avancée pour 1825 dans la chronologie (« de 6000 à 8000 habitants », p. 19) ne doit rien à l'escale de Beechey cette année-là ( $\ll 1500 »$, citation exacte p. 57), mais a été formulée pour une période plus ancienne par A. C. Eugène Caillot (1914 : 233), dont la bibliographie du catalogue ne cite aucun des ouvrages, malgré leur importance pour l'histoire de Mangareva. On ne peut croire qu'en préparant leur contribution (« Origine, peuplement et archéologie», pp. 52-57), Éric Conte et Patrick V. Kirch aient songé à minimiser ou à passer sous silence les recherches pionnières de leur collègue, doyen et ami Roger C. Green, qui continue d'étudier ses trouvailles de 1958 à Mangareva et, s'ils y font allusion sans le citer nommément, il faut l'attribuer aux contraintes qui leur ont été imposées. Seules échappent à ce défaut les descriptions des sculptures (pp. 22-44) par Catherine Orliac et, pour l'une d'entre elles, par Laurent Guillaut (d'après les obser- 